\title{
IGG4-RELATED DISEASE: THE PATH TO THE RHEUMATOLOGIST
}

Gizelle Gouvea Rezende ${ }^{1, \star}$, Viviane Queiroz de Oliveira Maia1', Danielly Dantas Pimentel', Viviane Alves Costa', Luciana Akita', lane Tamara Dondé1, Gustavo Roberto Lourenço', Juliana de Jesus Boscolo', Gustavo Resende Nora', Rafael Felipe Maciel Andrade ${ }^{1}$, Taisa Morete da Silva', Roberto Acayaba de Toledo ${ }^{1}$

1.Faculdade de Medicina de São José do Rio Preto, São José do Rio Preto (SP), Brazil.

*Corresponding author: gizellerezende@hotmail.com

\section{BACKGROUND}

IgG4-related disease (IgG4-RD) is a chronic inflammatory condition immune-mediated that can involve one or more organ systems, characterized by tissue infiltration with lymphocytes and lgG4-secreting cells into the plasma. There may be tumefactive lesions, fibrosis and a polyclonal lgG4-positive (IgG4+) plasma cell-enriched infiltrate. The classic IgG4-RD patient, unlike the case we will report, is usually a middle-aged to elder man. Inflammation and deposition of connective tissue in the affected sites can lead to organ dysfunction, organ failure and death, if left untreated. Serum IgG4 is usually elevated, otherwise the diagnosis requires histological confirmation. Glucocorticoids are first-line agents recommended for treatment, they present dramatic clinical results and often radiographic resolution.

\section{CASE REPORT}

M.L.S., a 30-year-old female, in ophthalmological follow-up of nodulation in topography of the lacrimal gland with diagnosis of chronic sclerosing dacryoadenitis confirmed by biopsy since 2010 and bilateral recurrence. In 2014, she presented weight loss and pain in the right hemithorax associated with massive pleural effusion, followed by a pulmonologist, who evidenced multiple pulmonary lymph nodes, biopsied and reported as reactional lymphoid hyperplasia of follicular and paracortical patterns. In 2019, hematology identified palpable lymph nodes in the cervical and inguinal regions and started investigating lymphoproliferative diseases, performing bone marrow and myelogram biopsies with normal results. The patient referred to Raynaud's phenomenon, diffuse arthralgia and morning stiffness. In face of this complaint, an evaluation by rheumatology department was requested. In addition, laboratory tests showed protein electrophoresis with polyclonal hypergammaglobulinemia; decreased C3 and C4; 1/640 homogeneous nuclear antinuclear antibody (ANA); blood count eosinophilia; high ESR and CRP. In 2020, based on previous diagnosis, clinical and laboratory history, the hypothesis of IgG4-RD was suggested. The investigation of lymphadenopathy was continued from imaging exams, chest and abdomen computed tomography (CT) showed mediastinal, hilar, retrocrural, axillary, retroperitoneal lymph nodes, heterogeneous renal parenchyma surrounded by non-calcified and hypocaptive soft tissue, suggestive of perirenal fibrosis. The diagnostic hypothesis of IgG4-RD was confirmed after inguinal lymph node biopsy (Fig. 1) submitted to immunohistochemistry (Fig. 2) and serum IgG4 dosage 50,800 (RV 30 to $2,010 \mathrm{mg} / \mathrm{dL}$ ).

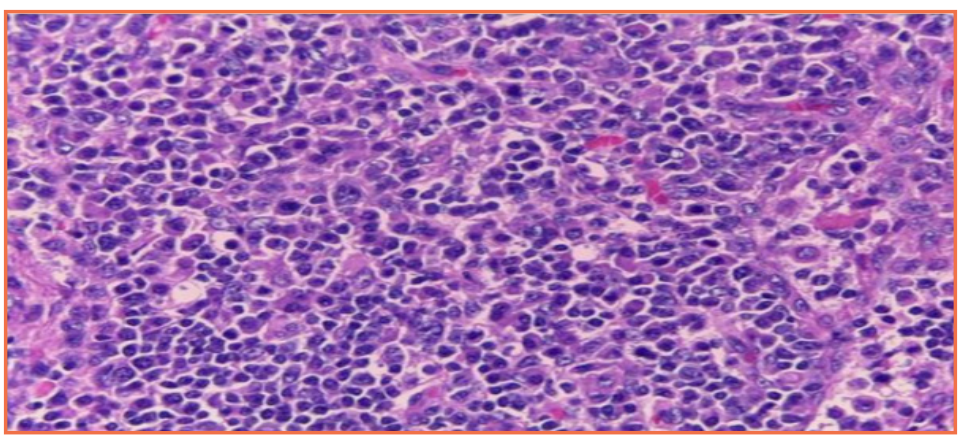

Figure 1. Presence of frequent plasma cells in interfollicular regions. 


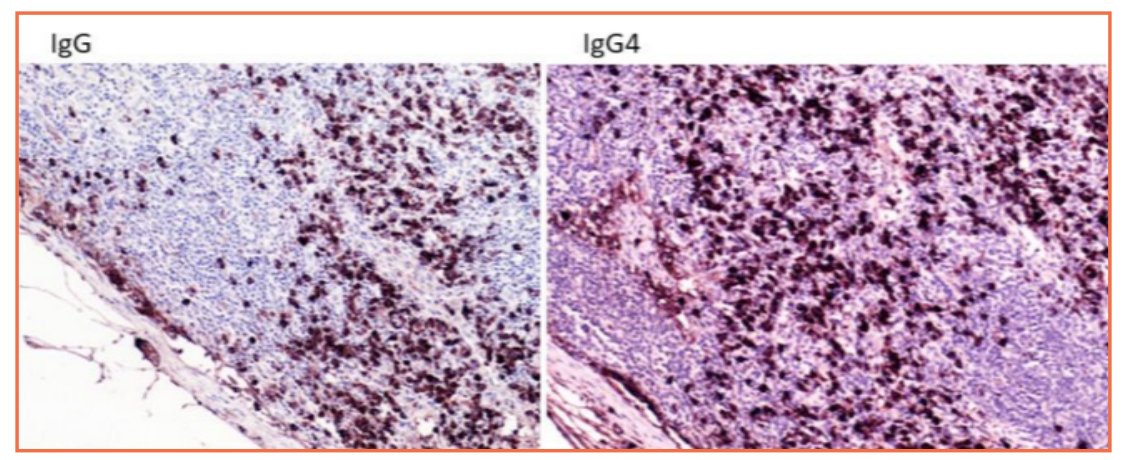

Figure 2. IgG showing moderate plasmacytosis, with most plasma cells having positive lgG4.

\section{CONCLUSION}

IgG4-related disease is rare, recently discovered and little known. The diagnosis usually takes years from initial symptoms to confirmation, countless times performed during advanced stages with involvement of multiple organs, limiting treatment and prognosis. 